\title{
Meeting Report: Translational Advances in Cancer Prevention Agent Development Meeting
}

\author{
Mark Steven Miller, ${ }^{1, *}$, Peter J. Allen ${ }^{2}$, Powel H. Brown ${ }^{3}$, Andrew T. Chan ${ }^{4}$, Margie L. Clapper ${ }^{5}$, \\ Roderick H. Dashwood ${ }^{6}$, Shadmehr Demehri ${ }^{7}$, Mary L. Disis ${ }^{8}$, Raymond N. DuBois ${ }^{9}$, Robert J. Glynn ${ }^{10}$, \\ Thomas W. Kensler ${ }^{11}$, Seema A. Khan ${ }^{12}$, Bryon D. Johnson ${ }^{13}$, Karen T. Liby ${ }^{14}$, Steven M. Lipkin ${ }^{15}$, \\ Susan R. Mallery ${ }^{16}$, Emmanuelle J. Meuillet ${ }^{17}$, Richard B.S. Roden ${ }^{18}$, Robert E. Schoen ${ }^{19}$, Zelton D. Sharp ${ }^{20}$, \\ Haval Shirwan ${ }^{21}$, Jill M. Siegfried ${ }^{22}$, Chinthalapally V. Rao ${ }^{23}$, Ming You ${ }^{24}$, Eduardo Vilar ${ }^{3}$, Eva Szabo ${ }^{25}$, \\ Altaf Mohammed ${ }^{1, *}$
}

${ }^{1}$ Chemopreventive Agent Development Research Group, Division of Cancer Prevention, National Cancer Institute, Rockville, Maryland, USA, ${ }^{2}$ Division of Surgical Oncology, Duke Cancer Institute, Durham, NC, USA, ${ }^{3}$ Department of Clinical Cancer Prevention, Division of Cancer Prevention and Population Sciences, MD Anderson Cancer Center, Houston, TX, USA, ${ }^{4}$ Clinical and Translational Epidemiology Unit, Massachusetts General Hospital, Boston, MA, USA, ${ }^{5}$ Cancer Prevention and Control Program, Fox Chase Cancer Center, Philadelphia, PA, USA, ${ }^{6}$ Center for Epigenetics \& Disease Prevention, Institute of Biosciences \& Technology, Texas A\&M Health Science Center, Houston, TX, USA, ${ }^{7}$ Department of Dermatology, Massachusetts General Hospital, Boston, MA, USA, ${ }^{8}$ Cancer Vaccine Institute, University of Washington School of Medicine, Seattle, WA, USA, ${ }^{9}$ Department of Biochemistry and Molecular Biology, Medical University of South Carolina, Charleston, SC, USA, ${ }^{10}$ Division of Preventive Medicine, Brigham \& Women's Hospital, Boston, MA, USA, ${ }^{11}$ Public Health Sciences Division, Fred Hutchinson Cancer Research Center, Seattle, WA, USA, ${ }^{12}$ Department of Surgery, Feinberg School of Medicine, Northwestern University, Chicago, IL, USA, ${ }^{13}$ Department of Microbiology \& Immunology, Medical College of Wisconsin, Milwaukee, WI, USA, ${ }^{14}$ Department of Pharmacology \& Toxicology, Michigan State University, East Lansing, Michigan; ${ }^{15}$ Division of Gastroenterology and Hepatology, Weill Cornell University, New York, NY, USA, ${ }^{16}$ Department of Oral and Maxillofacial Pathology, College of Dentistry, Ohio State University, Columbus, OH, USA, ${ }^{17} \mathrm{PH}$ Hsis Therapeutics, San Diego, CA, USA, ${ }^{18}$ Department of Pathology, Cancer Prevention and Control Program, Sidney Kimmel Comprehensive Cancer Center, Johns Hopkins University School of Medicine, Baltimore, MD, USA, ${ }^{19}$ Division of Gastroenterology, Hepatology and Nutrition, University of Pittsburgh School of Medicine, Pittsburgh, PA, USA, ${ }^{20}$ Department of Molecular Medicine, University of Texas Science Center at San Antonio, San Antonio, TX, USA, ${ }^{21}$ Department of Child Health and Molecular Microbiology and Immunology, University of Missouri, Columbia, MO, USA, ${ }^{22}$ Department of Pharmacology, Masonic Cancer Center, University of Minnesota School of Medicine, Minneapolis, MN, USA, ${ }^{23}$ Medical Oncology Center for Cancer Prevention \& Drug Development, Stephenson Cancer Center, University of Oklahoma Health Sciences Center, Oklahoma City, OK, USA, ${ }^{24}$ Department of Pharmacology and Toxicology, Cancer Center, Medical College of Wisconsin, Milwaukee, WI, USA, ${ }^{25}$ Lung and Upper Aerodigestive Cancer Research Group, Division of Cancer Prevention, National Cancer Institute, Rockville, MD, USA

Received March 4, 2021, Accepted March 5, 2021

Correspondence to Mark Steven Miller, E-mail: mark.miller5@nih.gov, https://orcid.org/0000-0003-1191-0977

Check for updates Altaf Mohammed, E-mail: altaf.mohammed@nih.gov, https://orcid.org/0000-0003-1058-6909

*These authors contributed equally to this work as co-correspondence authors. 
The Division of Cancer Prevention of the National Cancer Institute $(\mathrm{NCl})$ and the Office of Disease Prevention of the National Institutes of Health co-sponsored the Translational Advances in Cancer Prevention Agent Development Meeting on August 27 to 28 , 2020. The goals of this meeting were to foster the exchange of ideas and stimulate new collaborative interactions among leading cancer prevention researchers from basic and clinical research; highlight new and emerging trends in immunoprevention and chemoprevention as well as new information from clinical trials; and provide information to the extramural research community on the significant resources available from the $\mathrm{NCl}$ to promote prevention agent development and rapid translation to clinical trials. The meeting included two plenary talks and five sessions covering the range from pre-clinical studies with chemo/immunopreventive agents to ongoing cancer prevention clinical trials. In addition, two $\mathrm{NCl}$ informational sessions describing contract resources for the preclinical agent development and cooperative grants for the Cancer Prevention Clinical Trials Network were also presented.

Key Words Chemoprevention, Immunoprevention, Cancer vaccines, Clinical trials as topic

\section{INTRODUCTION}

The concept of cancer prevention has undergone a major paradigm shift over the last two decades. This is partially due to our understanding that the risk associated with long term treatment of the asymptomatic, low risk general population may cause more harm from agent toxicities than the preventive effects of the agents, as well as advances in precision medicine [1-4]. Primary cancer prevention is now thought to be best targeted to individuals identified to be at an increased risk of developing cancer as a result of inherited genetic polymorphisms (i.e., Lynch Syndrome, Familial Adenomatous Polyposis, Brca1/2 carriers) or exposure to causative environmental factors (i.e., current and former cigarette smokers). Of the examples just cited, lung, colon, and pancreatic cancer are three of the four leading causes of cancer deaths in both men and women in the United States [5]. The five-year overall survival rate for small cell lung cancer and pancreatic cancer patients is less than 20 and $10 \%$, respectively, making them high priorities for targeted prevention strategies as mandated in the Recalcitrant Cancer Research Act of 2012. Recent advances in the development of targeted chemopreventive and immunopreventive agents [4], as well as recent progress in the development of preventive vaccines $[6,7]$, hold significant promise for preventing or delaying the occurrence of cancer in high-risk patients, thereby reducing cancer-associated morbidity and mortality.

The Division of Cancer Prevention of the National Cancer Institute $(\mathrm{NCl})$ and the Office of Disease Prevention of the National Institutes of Health co-sponsored the Translational Advances in Cancer Prevention Agent Development Meeting on August 27 to 28, 2020. The main goals of this meeting were to foster the exchange of ideas and stimulate new collaborative interactions among leading cancer prevention researchers from basic and clinical research; highlight new and emerging trends in immunoprevention and chemoprevention as well as new information from clinical trials; and provide information to the extramural research community on the significant resources available from the $\mathrm{NCl}$ to promote prevention agent development and rapid translation to clinical trials. The meeting covered recent advances in new chemopreventive and immunomodulatory agent development, alternative dosing regimens, the development of preventive vaccines targeting neoantigens or oncogenic drivers of cancer initiation, and ongoing clinical trials of cancer preventive agents through presentations by 24 speakers. In addition, two $\mathrm{NCl}$ informational sessions describing contract resources for the PREVENT Preclinical Drug Development Program and cooperative grants for Cancer Prevention Clinical Trials Network (CP-CTNet) were also presented. A brief summary of the presentations highlighting major findings is reported.

\section{PLENARY SESSIONS}

Dr. DuBois' presentation "Inflammation and inflammatory mediators as targets for cancer prevention/interception", described the evidence for the link between inflammation and cancer that comes from epidemiologic and clinical studies showing that use of nonsteroidal anti-inflammatory drugs (NSAIDs) reduces the relative risk for developing colorectal cancer (CRC) by $40 \%$ to $50 \%$ and in some cases reduces cancer incidence. He presented new preliminary data showing that a COX-2 selective inhibitor or an prostaglandin E2 receptor 4 (EP4) antagonist for one of four prostaglandin E2 $\left(\mathrm{PGE}_{2}\right)$ receptors reduces intestinal adenoma burden accompanied with decreased programmed cell death protein 1 (PD1) expression in tumor-infiltrating $\mathrm{CD}^{+} \mathrm{T}$ cells and tumor-associated macrophages (TAMs) in in vitro and in vivo models. These novel suggest that $\mathrm{PGE}_{2}$ produced locally in the tumor microenvironment promotes tumor immune evasion by suppressing functions of tumor-infiltrating $\mathrm{CD}^{+} \mathrm{T}$ cells and TAMs via induction of PD-1 $[8,9]$.

Dr. Brown's talk "Novel Cancer Prevention Strategies in the Molecular Era", summarized the findings from several Phase III cancer prevention trials and the results of preclinical studies and early phase cancer prevention trials testing novel cancer prevention approaches with a focus on reducing toxicity. Novel interventions included low-dose tamoxifen therapy, topically applied tamoxifen, molecular targeted drugs inhibiting COX-2, mTOR, or PARP pathways, vaccines, checkpoint 
inhibitors, and cytokine inhibitors. These studies demonstrated that it is possible to prevent breast, colon, and lung cancer in animal models, and early-phase clinical trial results show promising results. However, toxicity remains a major issue and the challenge for the future will be to develop minimally toxic prevention strategies that will be acceptable to individuals at high-risk of cancer. Significant advances in targeting oncogenic driver molecules and in harnessing the immune system offer great promise to safely prevent many life-threatening cancers in the near future.

\section{SESSION 1: ADVANCES IN SMALL MOLECULE AGENT DEVELOPIMENT}

Dr. Margie Clapper's presentation "Estrogen Metabolism: A Target for Intervention in Non-Small Cell Lung Cancer", described potential mechanisms for the unique clinical and pathological features of NSCLC among never-smokers, where over $50 \%$ of those affected are females. Her group is the first to demonstrate that the human lung can extensively metabolize estrogen to several derivatives, including the putative carcinogen 4-hydroxyestrogen (4-OHE). The level of each parent estrogen and estrogen metabolite was significantly higher in females vs. males. 4-OHE, as a percentage of total estrogen, was elevated significantly in tumors vs. adjacent normal tissue from patients with NSCLC. Genetic and chemical inhibition of cytochrome P450 (CYP1B1), the enzyme responsible for 4-OHE production, in cultured cells led to reductions in cell motility and proliferation. Based on the high proportion of never-smokers among female lung cancer cases in Asia (60\% to $80 \%)$, the ability of Asian women to produce 4-OHE was evaluated. Urinary levels of 4-OHE (\% total) were significantly higher in Chinese American vs. Caucasian American women matched for age and body mass index, suggesting healthy Chinese women are high producers of 4-OHE. These findings, when combined with additional preclinical data, suggest estrogen metabolism represents a promising target for intervention in NSCLC.

Dr. Liby's talk "Targeting the Immune System for the Prevention of KRAS-Driven Cancers", described the role of combinations of rexinoids, selective agonists for retinoid $X$ receptors [10], and the synthetic oleanane triterpenoid [11] bardoxolone methyl, an activator of the cytoprotective Nuclear factor E2-related factor 2 (Nrf2) pathway, as attractive drugs for preventing lung and pancreatic cancer because they are active in preclinical models of lung and pancreatic cancer driven by Kras mutations. Both classes of drugs favorably altered immune cells populations, including reducing the number of immunosuppressive $\mathrm{Gr} 1+$ myeloid derived suppressor cells (MDSCs) in the pancreas of the LSL-Kras ${ }^{\mathrm{G} 12 \mathrm{D} /+} ; \mathrm{Pdx}-1-$ Cre (KC) or LSL-Kras ${ }^{\mathrm{G} 12 \mathrm{D} /+} ;$ LSL-Trp53 $^{\mathrm{R} 127 \mathrm{H} /+} ; \mathrm{Pdx}-1-C r e(\mathrm{KPC})$ mice. When tested for prevention of lung cancer, new rexinoids reduced both MDSCs and macrophages around lung tumors without elevating triglycerides, a known side effect of many rexinoids. Surprisingly, they found a higher tumor burden and a novel immune signature in the lungs and tumors of Nrf2 knockout (KO) mice compared to wildtype mice with lung cancer [12]. The numbers of tumor-promoting macrophages and MDSCs were elevated in the Nrf2 KO mice, while beneficial cytotoxic CD8+ T cells were decreased. These findings are consistent with data from lung cancer patients found in The Cancer Genome Atlas and suggest that activation of the Nrf2 pathway in immune cells can be used to prevent lung cancer.

Dr. Sharp's presentation "Cancer and Aging Prevention by mTOR Inhibition", described previous in the $\mathrm{Apc}^{\mathrm{Min} /+}$ mice model of familial adenomatous polyposis showing that chronic treatment of $\mathrm{Apc}^{\mathrm{Min} / \mathrm{+}}$ females with an enteric formulation of the anti-aging drug, rapamycin (eRapa), restored a normal lifespan through reduced polyposis and anemia prevention [13]. Lifespan extension by chronic rapamycin in wildtype UMHET3 mice is sex-dependent with females gaining the most benefit. Similar studies comparing male and female $\mathrm{Apc}^{\mathrm{Min} /+}$ mouse following chronic treatment with an eRapa-containing diet found that survival of males was greater than females in this setting. Immunohistochemistry assays of rpS6 phosphorylation showed that rapamycin reduction of mammalian target of rapamycin complex 1 (mTORC1) activity is most prominent in small intestine crypt Paneth cells. Chronic rapamycin also reduced crypt depths equally in both male and female $\mathrm{Apc}^{\mathrm{Min} /+}$ mice, consistent with reduced crypt epithelial cell proliferation. Chronic eRapa diet significantly extended lifespan of dextran sodium sulfate (DSS) treatments ( $\mathrm{Apc}^{\mathrm{Min} /+}$-DSS model) in both sexes by reductions in colon neoplasia and prevention of anemia. IHC analyses demonstrated that cells at the base of crypts had a prominent reduction in rpS6 phosphorylation relative to controls. Collectively, the data indicated that enteric rapamycin prevented polyposis in $\mathrm{Apc}^{\mathrm{Min} /+}$ mice of both sexes and delayed or prevented colon neoplasia in $\mathrm{Apc}^{\mathrm{Min} /{ }^{+}}$-DSS mice through inhibition of mTORC1 in small intestine Paneth cells and cells at the base of colon crypts, respectively, thereby significantly extending their life and health span [14].

Dr. Kensler's talk "Bringing Broccoli Sprouts (Sulforaphane) to Clinical Trials: Dose Matters", described his ongoing clinical trials with the chemopreventive agent sulforaphane. Sulforaphane is an inducer of glutathione S-transferases and other cytoprotective enzymes through activation of Nrf2 signaling. Translating this efficacy into the design and implementation of clinical chemoprevention trials, especially food-based trials, faces numerous challenges including the selection of the source, formulation, placebo, dose of the intervention material [15], and informative biomarkers [16]. Notable in the case of sulforaphane, selection of doses has been ill-informed by most pre-clinical studies. Randomized clinical trials to evaluate the effects of composition (glucoraphanin-rich vs. sulforaphane-rich or mixture beverages), formulation (beverage vs. tablet) and dose on the efficacy of these broccoli sproutbased preparations were carried out in Qidong, China, whose 
residents are exposed to moderately high ambient levels of air pollution during the winter months. Rapid and sustained, statistically significant increases in the levels of excretion of the glutathione-derived detoxication conjugates of benzene and acrolein were found in those receiving broccoli sprout beverages compared with placebo beverages which persisted throughout the intervention [17]. However, the dynamic range for enhancement of S-phenyl mercapturic acid excretion was quite limited, perhaps reflecting a limited capacity to modulate NRF2 in humans [18]. Improved and more consistent bioavailability is achieved with tablets containing glucoraphanin and myrosinase. Thus, interventions with broccoli sprout-based preparations enhance the detoxication of some airborne pollutants and may provide a frugal means to attenuate their associated long-term health risks.

\section{SESSION 2: ADVANCES IN IMMUNOMODULATORY AGENT DEVELOPMENT}

Dr. Siegfried's talk "STAT3 Decoy for Preventing Lung Cancer", described a novel inhibitor of the STAT3 pathway of the STAT-3 pathway. CS3D is a cyclic double-stranded DNA oligonucleotide decoy that mimics the STAT3 response element. CS3D competitively blocks the binding of activated STAT3 dimers to the promoter regions of STAT3 target genes, and also induces p-STAT3 degradation. Former smokers are at elevated lung cancer risk, and account for a large proportion of newly diagnosed lung cancer. Former smokers with airway dysplasia have received some benefit in randomized trials of chemoprevention, while active smokers have not. To date, prevention trials in lung cancer have only shown modest effects. There is a great need for more effective chemoprevention agents for the millions of former smokers at risk for lung cancer. Extending previous data obtained in xenograft models, her laboratory determined the chemoprevention efficacy of CS3D using the NNK-induced mouse model of lung cancer. Following a 1-week rest period to mimic smoking cessation, CS3D or CS3M was given as a short-term intermittent therapy via intravenous injection $(5 \mathrm{mg} / \mathrm{kg}$ three times per week for 8 weeks). Compared to CS3M mutant inactive version, CS3D blocked formation and progression of airway preneoplasia. CS3D also reduced both incidence and size of lung tumors that arose over time, and induced apoptosis in the airways, while showing no discernable toxicity. Efficacy was associated with reduction of p-STAT3 protein detected by immunohistochemistry in dysplastic airway lesions, lung adenomas, and lung adenocarcinomas from these mice. Phospho-STAT3 protein was reduced both during and after treatment with CS3D, remaining suppressed 8 weeks after the end of the treatment course. Other pathways downregulated by CS3D were NF- $\mathrm{B}$, COX-2, IL-6, and VEGF. CS3D also produced a less immunosuppressive microenvironment in the lungs, with fewer M2 macrophages and MDSC cells.
No signs of toxicity were detected during therapy and no organ abnormalities were detected at necropsy. These results suggest that blocking STAT3 may be a useful strategy for lung cancer prevention and may involve both inhibition of oncogenic signaling and enhanced anti-tumor immunity.

Dr. Glynn's presentation "Mediation Analysis to Elucidate the Role of Inflammation Reduction in Cancer Prevention: Exploratory Findings from the CANTOS Trial", elucidated the results obtained from the Canakinumab Anti-inflammatory Thrombosis Outcomes Study (CANTOS). CANTOS randomized 10,061 individuals with prior myocardial infarction, who were free of previously diagnosed cancer and had concentrations of high-sensitivity C-reactive protein (hsCRP) $\geq 2 \mathrm{mg} / \mathrm{L}$, to either placebo or one of three active doses of canakinumab, a human anti-IL-1 $\beta$ monoclonal antibody $(50 \mathrm{mg}, 150 \mathrm{mg}$, or $300 \mathrm{mg}$ subcutaneously every 3 months). During median follow-up of 3.7 years, incident lung cancer occurred in 129 and fatal cancer in 196 subjects, with relative hazards of 0.55 (95\% confidence interval [95\% Cl]: 0.39 to 0.78 ) and 0.71 (95\% Cl: 0.53 to 0.94 ) in those receiving active canakinumab versus placebo, respectively $[19,20]$. For both outcomes, each of six measures of achieved level or change in an inflammatory biomarker showed evidence of significant mediation, ranging from $75 \%$ of the observed effect of canakinumab on fatal cancer mediated through continuous level of log (hsCRP) at 3 months to $33 \%$ of the observed effect of canakinumab on incident lung cancer mediated through achieving a level of hsCRP at 3 months $<2 \mathrm{mg} / \mathrm{L}$ [21]. For incident lung cancer, change in IL- 6 (mediating $51 \%$ of the observed canakinumab effect) had the strongest mediation effect. We saw little evidence for a direct effect of canakinumab outside the pathway of measured inflammation reduction. Measured changes in inflammatory markers strongly mediated reductions in incident lung cancer and fatal cancer associated with canakinumab treatment. Identification of powerful mediating variables can help target the most likely responders soon after treatment initiation.

Dr. Shirwan's talk "CD137 immune checkpoint pathway as an effective target for cancer immunoprevention", talk described his laboratory's studies the immune checkpoint stimulator CD137 pathway. The 4-1BB pathway plays a paramount role in the expansion of CD8+ T cells, acquisition of effector function, survival, and establishment of longterm memory that are important to cancer eradication and control of recurrence. The natural CD137 ligand lacks the costimulatory function as a soluble protein; thus, his laboratory generated an oligomeric form of the ligand, SA-4-1BBL [22], and demonstrated that it has better costimulatory activity and safety profile than agonistic CD137 Abs [23], which are presently being evaluated in cancer immunotherapy trials. Treatment with SA-4-1BBL as a single agent conferred protection against subsequent challenge with multiple tumor types, including melanoma, lung, lymphoma, and triple-negative breast cancer. Importantly, the immunoprevention was 
a bona fide feature of SA-4-1BBL as agonistic Abs to CD137 receptor had no impact on the tumor growth [24]. The cancer immunoprevention effect of SA-4-1BBL operated through an innate immune surveillance mechanism that evolved within three weeks of treatment, lasted for months, and did not involve CD8+ $T$ cells. SA-4-1BBL was also effective in controlling post-surgical recurrence against various tumors. The ability of an immune checkpoint stimulator as a single agent to train the immune system for long-lasting broad protection against many tumor types is exciting conceptually and sets the stage for a cancer immunoprevention modality that does not require tumor-specific antigens.

Dr. Demehri's presentation "Epithelium-Derived Alarmins Role in Breast Cancer Immunoprevention", described their studies to determine the benefit of activating the immune system during the early phases of breast cancer development to prevent cancer development and recurrence. The goal is to develop an effective approach to activate the patients' own immune system against early breast precursor lesions, which may yield a lasting memory that can prevent breast cancer development and recurrence in high-risk populations. His laboratory demonstrated that a skin-derived alarmin cytokine, thymic stromal lymphopoietin (TSLP), suppresses the early stages of breast cancer development [25]. The follow-up studies have revealed that $\mathrm{CD}^{+} \mathrm{T}$ helper 2 (Th2) are required and sufficient to deliver the tumor-protective effect of TSLP on breast cancer. Further, this antigen-specific immunity is mediated through terminal differentiation of breast tumor cells, which lasts long after TSLP induction is stopped. These findings establish a foundation for the use of the alarmin cytokines in blocking breast cancer development and provide novel therapeutic targets for breast cancer immunoprevention.

\section{SESSION 3: ALTERNATIVE DOSING AND COMBINATION STRATEGIES TO REDUCE TOXICITY}

Dr You's talk "Chemoprevention of Aerosolized Let-7 MicroRNA Mimic in a Mouse Lung Cancer Model", described the delivery of microRNA (miRNA) via inhalation as a potential strategy for lung cancer prevention in high risk individuals. Previous studies have shown that intratracheal or intranasal exposure of genetically engineered mice to viral vectors expressing let-7 miRNA resulted in a reduced lung tumor burden. Extending these studies to the benzo(a)pyrene-induced $\mathrm{A} / \mathrm{J}$ mouse lung tumor model, his laboratory treated mice with an aerosolized let-7b miRNA formulation. The particle size of the let-7b miRNA aerosol has been systematically characterized as particles with sizes between 1.9 to $35 \mathrm{~nm}$ (average size of $29.0 \mathrm{~nm}$ ). Following the aerosol formulation evaluation, the biodistribution of let-7b in the lung by tail vein injection and aerosolized delivery were compared. The distribution of the aerosolized let-7b produced higher levels of the
miRNA in the lung compared with systematic delivery. The aerosol delivery of let-7b had no effect on animal body weight nor were there any other signs of toxicity. The development of an aerosolized let-7b formulation inhibited $\mathrm{B}[\mathrm{a}] \mathrm{P}$-induced lung tumor volume by $72 \%$ in $\mathrm{A} / \mathrm{J}$ mice. Preliminary scRNAseq analyses showed that let-7b targets both tumor cells and immune cells in the tumor microenvironment. Collectively, these findings show that aerosolized miRNA is a promising approach for lung cancer prevention.

Dr. Mallery's presentation "Oral Cancer Chemoprevention by Local Delivery", described the use of local chemoprevention strategies for treatment of oral squamous cell carcinoma. By direct application to the treatment site, local delivery formulations demonstrate a pharmacologic advantage i.e., achieve therapeutic levels at the target with negligible systemic adverse effects. While direct application of a bio adhesive gel that contained $10 \% \mathrm{w} / \mathrm{w}$ of freeze dried black raspberries (BRB) provided positive clinical data that included significant reduction in histologic grade, lesion size, and loss of heterozygosity at putative tumor suppressor gene loci, not all patients responded uniformly and, as a natural product, BRB cannot be standardized for sustained, high level production. Thus, the synthetic derivative of vitamin A fenretinide (4HPR) was selected. As a highly hydrophic drug, 4HPR delivery to a saliva-rich site like the mouth is challenging. $A$ Tegaderm-backed patch that incorporated 4HPR solubility enhancing agents (sodium deoxycholate and Tween-80) and permeation enhancers (menthol, propylene glycol) successfully delivered therapeutically relevant $4 \mathrm{HPR}$ levels to rabbit oral mucosa. Furthermore, no 4HPR was detected in the sera (LC-MS/MS), and no clinical adverse events such as a contact mucositis were noted. Consistent with 4HPR's growth modulatory effects, treated mucosa demonstrated a slight increase in the outer cornified layer (lower 4HPR levels) and increased apoptosis (higher 4HPR levels). An additional positive effect was the increase in surface epithelial levels of the Phase II detoxification enzyme UGT1A1. Work is ongoing to refine the clinical patch formulation to be used in the upcoming clinical trial.

Dr. Rao's presentation "Safer Chemopreventive Approaches to Colonic Adenoma Prevention", described studies to reduce the GI toxicity and unwanted side effects of continuous treatment regimens employing nonsteroidal anti-inflammatory drugs for colon cancer prevention. Using the azoxymethane treated F344 male rat model, his laboratory tested different dosing regimens of Naproxen and Aspirin to prevent colon cancer formation. Rats were fed diets containing Naproxen (200 and 400 ppm) or Aspirin (700 and 1,400 ppm) either continuously, 1 week on/ 1 week off, or 3 weeks on/3 weeks off, or Aspirin (2,800 ppm) 3 weeks on/3 weeks off. Dietary administration of Naproxen and Aspirin did not show any overt toxicities. Both NSAIDs exhibited dose response effects and demonstrated inhibition of invasive colon carcinoma with different treatment regimens. Both agents showed signifi- 
cant modulation of proliferative (PCNA, p21) and apoptotic markers ( $p 53$, Casp3) in colonic tumors. Transcriptomic data revealed that proinflammatory cytokines, particularly interleukins and metalloproteases, were significantly reduced in tumors of rats exposed to Aspirin or Naproxen [26]. Additional approaches tested showed treatment with the dual cyclooxygenase (COX)/5-lipoxygenase (LOX) inhibitor Licofelone resulted in superior efficacy compared to a COX-2 inhibitor (celecoxib) alone in suppressing adenoma progression to adenocarcinoma. LFA-9, a dual inhibitor of the prostaglandin E synthase-1 5-LOX enzymes which does not affect PGI2 was found to also exhibit strong efficacy against colon tumor formation in both mice and rat colon cancer models. Overall, these results suggest that intermittent dosing with Naproxen or Aspirin; targeting COX/5-LOX and mPGES-1/5-LOX are considered to be safer approaches to prevent colon adenoma progression to adenocarcinoma.

Dr. Meuillet's talk "A dual AKT/PDPK1 inhibitor for actinic keratosis and skin cancer prevention in immunocompromised individuals" described her company's ongoing study utilizing a dual kinase inhibitor. Epicutaneous (topical) therapy with molecularly targeted agents is an attractive modality for treatment of basal cell carcinoma (BCC), early UVB induced squamous cell carcinoma (SCC) and cutaneous metastatic disease (CMD) associated with metastatic breast cancer and Actinic Keratoses, especially in immunocompromised subjects (organ transplants, HIV, other cancers etc.). There is evidence that Akt and PDKP1 play complimentary yet independent roles in phosphatidylinositol-3 kinase (PI3K) pathway signaling associated with driving the growth of $B C C$, UVB induced SCC, and breast CMD. Both Akt and PDPK1 possess a pleckstrin homology $(\mathrm{PH})$ domain, a highly conserved three-dimensional superfold with a high affinity for binding phosphatidylinositol-3-phosphates, causing Akt and PDPK1 to translocation to the plasma membrane where Akt is activated. Through reiterative molecular docking and structure refinement using a proprietary computational platform, PHUsis has identified PHT-427 as an agent that binds to the $\mathrm{PH}$ domains of Akt and PDPK1, inhibiting their activity. PHT-427 has antitumor activity when administered orally but importantly also following epicutaneous administration. Evaluation of the antitumor potential of topical PHT-427 against CMD in an intradermal breast xenograft model and in an early UVB induced skin cancer model in mice show promising effects of topical PHT-427 in cancers with skin involvement. Thus, topical application of PHT-427 can deliver active drug to skin and tumor, inhibiting AKT and PDPK1, both of which drive the PI3K pathway important in UVB induced SCC, breast CMD, and AKT in immunocompromised subjects, with significant inhibition of tumor growth without adverse effects on normal skin.

Dr. Dashwood's presentation "Optimization of erlotinib plus sulindac dosing regimens for intestinal cancer prevention in an Apc-mutant model of Familial Adenomatous Polyposis
(FAP)", reported that sulindac plus erlotinib (SUL+ERL) had good efficacy in the polyposis in rat colon (Pirc) model, which mimics several features of human FAP [27-32]. Phosphorylation of extracellular signal-related kinase ( $p$ Erk) was inhibited in colon polyps for up to 10 days after discontinuing ERL + SUL administration. In a study lasting 16 weeks, significant reduction of colon and small intestine tumor burden was detected, especially in rats given 250 ppm SUL in the diet plus once-a-week intragastric dosing of ERL at 21 or $42 \mathrm{mg} /$ $\mathrm{kg}$ body weight (BW). A long-term study further demonstrated antitumor efficacy in the colon and small intestine at 52 weeks, when 250 ppm SUL was combined with once-a-week intragastric administration of ERL at 10, 21 or $42 \mathrm{mg} / \mathrm{kg} \mathrm{BW}$. Tumor-associated matrix metalloproteinase-7 (Mmp7), tumor necrosis factor (Tnf) and early growth response 1 (Egr1) were decreased at 16 weeks by ERL + SUL, and this was sustained in the long-term study for Mmp7 and Tnf. The optimal dose combination of ERL $10 \mathrm{mg} / \mathrm{kg}$ BW plus $250 \mathrm{ppm}$ SUL lacked toxicity, normalized hematocrit/organ weights, inhibited tumor-associated molecular biomarkers, and exhibited effective antitumor activity. They concluded that switching from continuous to once-per-week ERL, given at one-quarter of the current therapeutic dose, will exert good efficacy with standard of care SUL against adenomatous polyps in the colon and in the small intestine, with clinical relevance for FAP patients before or after colectomy.

\section{SESSION 4: EMERGING VACCINES FOR CANCER PREVENTION}

Dr. Lipkin's talk "Frameshift neoantigen vaccination prevent Lynch syndrome mouse model intestinal cancer", focused on microsatellite-unstable (MSI) cancers occurring in the context of Lynch syndrome. These tumors elicit pronounced tumor-specific immune responses directed against frameshift peptide (FSP) neoantigens, which result from mismatch repair (MMR) deficiency-induced insertion/deletion mutations in coding microsatellites (cMS). Results from a recently completed clinical phase I/Ila trial successfully demonstrated safety and immunogenicity of an FSP neoantigen-based vaccine in MSI colorectal cancer patients (Clinical trial number: NCT01461148) [33]. The vaccine was safe and induced robust cellular and humoral immune responses in all vaccinated patients. To establish a preclinical mouse model, a systematic database search was performed to identify cMS sequences in the murine genome. Subsequently, intestinal tumors obtained from Lynch syndrome mice (Msh2 $2^{\text {floxflox }} \mathrm{VpC}^{+/+}$) were evaluated for mutations affecting these candidate microsatellites [34]. Thirteen candidate cMS were detected that presented with a mutation frequency of $15 \%$ or higher. Epitope prediction using the netMHC4.0 algorithm was performed, and the ten most promising FSP neoantigens were synthesized. Immunogenicity was evaluated after vaccination of C57BL/6 mice using IFN-gamma ELISpot. Four FSP neoantigens derived from 
cMS mutations in the genes Nacad, Maz, Xirp1, and Senp6 elicited strong antigen-specific cellular immune responses. CD4-specific T cell responses were detected for Maz, Nacad, and Senp6 and CD8-positive T cells were detected for Xirp1 and Nacad. Vaccination with peptides encoding these four intestinal cancer FSP neoantigens promoted anti-neoantigen immunity, reduced intestinal tumorigenicity and prolonged overall survival $(P<0.01)$. Additionally, NSAIDs, which have chemopreventive efficacy for Lynch syndrome [35], increase $T$ cell immunity against neoantigens, thus supporting the further development of vaccination strategies for preventing cancers associated with Lynch syndrome.

Dr. Robert E. Schoen's presentation, "Randomized, Double-Blind, Placebo-Controlled Trial of MUC1 Vaccine in Patients with Newly Diagnosed Advanced Adenoma of the Colon", described immunoprevention via targeting antigens aberrantly expressed on CRC and its precursor, adenomas. His laboratory performed a double blind, randomized trial of a 100 aa peptide MUC1 vaccine admixed with an adjuvant TLR-3 agonist, polyICLC, vs. placebo in individuals with newly diagnosed advanced adenomas. Immunogenicity, measured by anti-MUC1 IgG at 12 weeks and immune memory at 1 year, and adenoma recurrence were assessed. Following vaccine or placebo administration at $0,2,10$, and 52 weeks, an anti-MUC1 IgG ratio of $\geq 2.0$ at 12 weeks (week 12/week 0 ) and at 55 weeks (week 55/week 52) was defined as a positive immune response. Adenoma recurrence was determined at colonoscopy $\geq 1$ year from initial vaccination from 103 subjects randomized at 6 centers. Subjects had a mean age of $59.4 \pm 7.0$ years, $62.1 \%$ were male, $88.3 \%$ were white, and $18.4 \%$ Hispanic, with no significant difference by arm. 13/52 (25.0\%) MUC1 vaccine recipients had a week 12/ week 0 ratio $\geq 2.0$, (range 2.9 to 17.3 ) vs. $0 / 50$ in the placebo group (1-sided Fisher's exact $P<0.0001$ ). 17/51 (33.3\%) MUC1 vaccine recipients had a week 55/week 52 ratio $\geq 2.0$ vs. $2 / 44(4.5 \%)$ placebo (1-sided Fisher's exact $P=0.0003)$. Eleven of the $13(84.6 \%)$ responders at week 12 responded at week 55/week 52 and were classified as immune responders. The mean time (SD) to follow up colonoscopy from initial vaccination was 886.1 days (248.9) for MUC1 vs. 923.0 (258.7) in the Placebo group $(P=0.36)$. In those receiving MUC1 vaccine, adenoma recurrence was observed in 27/48 $(56.3 \%)$ vs. $31 / 47(66.0 \%)$ receiving placebo $(P=0.22)$. In immune responders, adenoma recurrence was 3/11 (27.3\%) vs. $51 / 78(65.4 \%)$ in non-responders $(P=0.02)$. Thus, only vaccine recipients developed a positive anti-MUC1 lgG immune response. MUC1 vaccine recipients had a reduced, but non-statistically significant, $10 \%$ lower adenoma recurrence rate. In vaccine recipients who developed a positive anti-MUC1 lgG immune response, there was a statistically significant $38 \%$ reduction in adenoma recurrence. There was no significant toxicity to the MUC1 vaccine compared to placebo, other than increased injection site reactions. MUC1 vaccine is a promising approach to $\mathrm{CRC}$ prevention but efforts to im- prove the immune response to vaccine are needed. .

Dr. Roden's talk "Development of vaccines for broad protection against, and elimination of, HPV infection", described clinical studies with a candidate therapeutic and preventive HPV vaccine, pNGVL4a-CRTE6E7L2 (CRTE6E7L2), which comprises a DNA vector encoding the heat shock protein calreticulin fused genetically with HPV16 E6 and E7 (that are obligately expressed in HPV malignancies) as well as the L2 capsid protein (a broadly protective antigen). Fusion with calreticulin (CRT) profoundly enhances the potency of DNA vaccines in generating HPV antigen-specific CD8+ T cell mediated immune responses even in CD4-depleted animals. In addition, vaccination with the CRTE6E7L2 DNA vaccine induces both L2-specific neutralizing antibodies and protection from experimental vaginal challenge. DNA was produced for a clinical study and good laboratory practice (GLP) preclinical testing supported by the NCI PREVENT program. Although DNA vaccines are relatively safe and well suited for multiple administrations, they generally exhibit suboptimal immunogenicity when administered by conventional intramuscular needle injection, likely reflecting inefficient host cell transduction. His group has previously shown that electroporation is a much more effective DNA vaccine administration method to generate HPV-specific CD8+ T cell immune responses as compared to conventional intramuscular injection or epidermal delivery via gene gun. Thus, the goal of their recently opened NCI SPORE-funded Phase I clinical study (NCT04131413) is to use the Ichor TriGrid ${ }^{\mathrm{TM}}$ Delivery System Electroporation Device, which has been used in multiple clinical trials, for intramuscular administration of the CRTE6E7L2 DNA vaccine at escalating doses in both HIV- and HIV+ patients with HPV16-associated high-grade cervical intraepithelial neoplasia grades 2 and 3 (CIN2/3), and to examine the safety, virologic, and disease outcomes. This study aims to develop a new immunotherapy for the treatment of HPV-associated high-grade squamous intraepithelial lesions in both HIV- and HIV+ patients that also broadly prevents acquisition of new HPV infections.

Dr. Johnson's presentation "Development of a KRAS Preventive Vaccine", focused on a vaccine to prevent lung cancer which continues to be a major cause of cancer mortality worldwide. KRAS mutations can occur in up to one third of lung adenocarcinomas. To date, targeted therapies to mutant $K R A S$ have been challenging. Through a collaborative effort, his group is examining whether vaccines can be used to target mutant KRAS by mobilizing the immune system. Unfortunately, treatment of active malignancies poses many challenges to vaccines including the potent immune suppression that typically occurs. Due to these challenges, using vaccines to prevent the development of cancer could prove to be more efficacious. They developed a multi-peptide KRAS vaccine formulated with four peptides that had $100 \%$ homology between human and mouse KRAS. The vaccine was designed using $\mathrm{MHC}$ Class II binding algorithms, and peptides were 
screened for promiscuity across multiple MHC alleles. Using an inducible KRAS mouse model, they demonstrated that the multi-peptide vaccine was able to significantly reduce tumor burden when administered prior to induction of mutant KRAS expression. Anti-tumor efficacy correlated with induction of a $T$ cell immune response to the individual KRAS peptides. More recent data from their laboratory suggests that combining the KRAS vaccine with other immune-modulating therapies could further improve vaccine efficacy. With its direct translatability to humans, their preclinical data makes a compelling case for testing the multi-peptide KRAS vaccine in clinical trials as an immune-preventive agent for lung cancer $[36,37]$.

Dr. Disis' talk "Vaccines targeting cancer initiation associated proteins", illustrated a novel method for generating Class II (CD4) specific Type I immune responses targeting non-mutated tumor associated proteins. Type I CD4+ T-cell responses, Th1, can significantly alter the immune microenvironment to support a dominant cytotoxic T-cell response and epitope spreading, which is a broadening of the immune response to multiple antigens in the tumor. CD4 T-cell epitopes can be identified which bind with high affinity across multiple HLA-DR alleles (promiscuous epitopes) to create a universal vaccine which can be processed by diverse HLA. Appropriately primed CD4 T-cells establish immunologic memory which is essential for any cancer prevention vaccine. To create Th1 selective vaccines, epitope identification using web-based algorithms is followed by functional screening to identify Th2 or T-regulatory cell inducing epitopes, subsequently editing those epitopes from the vaccine sequence [38]. The strategy is now being applied to the development of multi-antigen vaccines for cancer prevention. One approach was to target immunogenic proteins expressed in the breast cancer stem cell. A multi-antigen polyepitope Th1 selective vaccine targeting immunogenic proteins associated with breast cancer stem cells to determine whether immunization could prevent the development of breast cancer in middle aged TgMMTV-neu mice. The multi-antigen vaccine prevented tumor growth in $50 \%$ of mice. Vaccination increased the influx of T beta + CD4 T-cells and CD8 T-cells to the tumors of transgenic mice [39]. A phase I clinical trial of STEMVAC was recently completed using the multi-antigen vaccine at three different dose levels in patients with advanced stage breast cancer. The vaccine was safe and immunogenic and induced only antigen specific Th1 without augmentation of Th2 or T-regulatory cells. These data lay the foundation of using STEMVAC to prevent the development of breast cancer in genetically high-risk individuals. The second example was a multi-antigen Th1 selective vaccine targeting colorectal cancer. The antigens consisted of proteins that were highly expressed in adenomas and that expression was conserved through the development of colorectal carcinoma [40]. The vaccine was combined with NSAID administration in APC min mice with treatment starting at 6 weeks of age. Animals receiving vaccine alone showed a $33 \%$ inhibition of polyp formation while naproxen alone showed $54 \%$ inhibition $(P<$ 0.0001 ) compared to adjuvant alone. Combination treatment demonstrated significantly greater inhibition of polyps than either modality $(P<0.001), 81 \%$ inhibition vs. adjuvant.

\section{SESSION 5: CANCER PREVENTION CLINICAL TRIALS}

Dr. Allen's presentation “Chemoprevention for Pancreatic Cancer", focused on understanding and managing high-risk precursor lesions of pancreatic cancer. In patients diagnosed prior to the development of identifiable metastatic disease (as occurs in $<15 \%$ of all pancreatic cancer patients) and are treated with resection and adjuvant systemic therapy, the probability of long-term survival is quite low. These patients, with the earliest lesions that can be identified by either cross-sectional imaging or endoscopy, experience dismal five-year survival rates of between $10 \%$ to $25 \%$. Intraductal papillary mucinous neoplasms (IPMN) of the pancreas represent the only radiographically identifiable precursor lesion of pancreatic cancer. This pathway of progression to pancreatic cancer is believed to represent between $20 \%$ to $30 \%$ of pancreatic cancer and currently represents the only identifiable lesion for which intervention can lead to cure. It is known that patients who undergo partial pancreatectomy for IPMN have an increased risk of developing cancer in the pancreatic remnant. These patients represent a very high-risk group for progression to pancreatic cancer as $7 \%$ to $15 \%$ of these patients will develop pancreatic cancer in their pancreatic remnant over a 4 to 5 -year time period. In addition, up to $25 \%$ of these patients will develop radiographic signs of progression over the same time period. Pre-clinical models of pancreatic cancer have found that NSAID agents such as sulindac to be effective at decreasing progression to pancreatic cancer. Data generated within from his laboratory has found a distinct association between inflammation and IPMN progression. Thus, anti-inflammatory strategies are applicable and promising for prevention of progression in IPMN patients. There is preliminary human evidence that sulindac may have efficacy in preventing radiographic progression in IPMN. Because of these findings, his research group has just launched the first randomized placebo-controlled trial to evaluate the ability of sulindac to prevent progression in patients with IPMN.

Dr. Vilar's talk "Cancer immune-interception for Lynch Syndrome", began by noting that an immunoprevention approach to Lynch syndrome (LS) is supported by multiple lines of evidence, including the identification MMR-deficient histologically normal appearing colon crypts as the earliest definable abnormality in pre-neoplastic colorectal epithelium in LS. Recent work by his group provided evidence of a robust immune activation signature in LS adenomas regardless of their mutation burden. By further characterizing the immune signature of LS adenomas, a global enrichment for $\mathrm{CD}^{+} \mathrm{T}$ 
cells and $\mathrm{FOXP3}^{+}$regulatory $\mathrm{T}$ cells was observed in the subset of patients with high mutation burdens. Moreover, there was up-regulation of both pro-inflammatory cytokines (IL12A) and checkpoint blockade (IFNG, CD274/PD1, and LAG3) [33]. These findings correlate well with the known biology and clinical significance of immune activation in carcinomas. With respect to immunomodulatory agents, NSAIDs inhibit COX2 and the downstream production of pro-tumorigenic prostaglandins that promote local inflammation. Prior work has shown that NSAIDs, more specifically aspirin, are associated with a modest but reliable chemopreventive benefit to reduce the risk of LS-related cancers after a continuous exposure of at least two years of duration to high doses (600 mg) [35]. His laboratory has extended these studies in an $\mathrm{NCl}$-sponsored Phase IB clinical trial to identify the immunomodulatory mechanism of another NSAID, naproxen sodium, when used for chemoprevention in LS carriers at two different doses (200 and $400 \mathrm{mg}$ ). Both doses of naproxen modulated the production of prostaglandins and also induced an activation of the immune resident cells in the colorectal mucosa [33], thus pointing towards its potential use as a modulator to be combined with upcoming vaccination strategies for LS cancer prevention [41].

Seema A. Khan's presentation, "Local transdermal drug delivery to the breast for cancer prevention", initially described the poor acceptance of oral endocrine therapy for breast cancer prevention, necessitating the development of alternative approaches. The reluctance of eligible high-risk women to accept oral endocrine agents is based, to a large extent, on concerns about systemic toxicity. One such alternative is the use of transdermal formulations of effective drugs, such as 4-hydroxytamoxifen (4-OHT) an active metabolite of oral tamoxifen. This has been shown to penetrate breast skin, with good retention in the breast and low systemic exposure; and to be as effective as oral tamoxifen in reducing cell proliferation of invasive and in-situ malignancy. However, several questions remain regarding the distribution of drug through the breast, the kinetics of clearance though the breast, and optimal frequency of dosing. Recent data from their group show that orally and transdermally administered drug is distributed similarly though the breast, but further optimization of doses, schedules, and transdermal formulations is needed. Ongoing randomized Phase II trials using 4-OHT gel will add weight to existing data, and provide a rationale to develop a Phase III trial of this agent, comparing it to oral endocrine therapy of choice, with the major endpoints being reduction in risk of new breast events, and reduction in breast density. Other agents are also under development, notably topical endoxifen, which may be more effective than topical 4-OHT, and topical bexarotene, which is already in use for cutaneous T-cell lymphoma, but safety and efficacy in the breast remains to be demonstrated. Other possibilities include topical NSAIDs (diclofenac is already available and approved for analgesia) and progesterone receptor antagonists, as well as small molecules such as lapatinib. For these, it may be necessary to involve nanoscience approaches, which are the next step in transdermal delivery development.

Dr. Chan's talk "Novel Strategies for Aspirin Prevention of Colorectal Cancer", noted the consistent experimental and epidemiologic evidence demonstrating that aspirin is associated with a lower risk of colorectal cancer. In 2016, the US Preventive Services Task Force updated its primary prevention guidelines to recommend low-dose aspirin (81 $\mathrm{mg} /$ day) for chronic disease prophylaxis, including colorectal cancer prevention, among US adults between ages 50 to 59 , and possibly ages 60 to 69 , with a greater than $10 \%$ tenyear risk of cardiovascular events. Despite this advance, there remains uncertainty about aspirin's risk-benefit profile in many populations, especially older adults. His group has led several studies into the mechanistic basis of aspirin's anti-cancer effect that culminated in the successful completion of the NCl-supported ASPirin for the REDuction of Colorectal Cancer Risk (ASPIRED) randomized placebo-controlled trial (RCT) which showed that aspirin reduces urinary prostaglandin metabolites, a validated biomarker of colorectal neoplasia [42]. His group also led an analysis of the Aspirin to Prevent Events in the Elderly (ASPREE), an RCT of aspirin in 19,114 apparently healthy older adults aged $65+$. Surprisingly, aspirin was associated with increased all-cause mortality that was driven primarily by cancer deaths that was not accompanied by an increase in cancer incidence over 4.7 years [43]. These results underscore our limited understanding of the influence of age on the biology of cancer and the context-dependent mechanisms by which aspirin may influence initiation, growth, and spread of cancer. Further investigation into potential age-dependent mechanisms of aspirin may lead to mechanistic biomarkers to improve risk-stratification for chemoprevention.

\section{NCI INFORMATION SESSIONS}

Drs. Miller and Szabo described resources available to the extramural community from the Division of Cancer Prevention in their talks "NCI PREVENT Preclinical Drug Development Program and Overview of the Cancer Prevention Clinical Trials Network (CP-CTNet)", respectively. Using a contract mechanism, the former program supports preclinical development of agents from initial efficacy studies through IND-enabling toxicology. The latter program supports clinical trials via a cooperative grant mechanism including providing regulatory and agent acquisition/distribution support. Information on these resources can be obtained at the Division of Cancer Prevention website: https://prevention.cancer.gov/home.

\section{ACKNOWLEDGMENTS}

Drs. Miller and Mohammed would like to thank our Staff Assistant, Ms. Wosene Asefa, for her outstanding assistance 
with the preparations for and organization of the meeting.

Dr. Allen was supported by $\mathrm{NCl}$ grant R01 CA235677. Dr. Brown was supported by Contracts HHSN261201500018I and 75N91019D00021 from the NCI PREVENT Program. Dr. Chan was supported by NIH grants R01CA137178, R01CA202704, R35CA253185, and U19AG062682. Dr. Clapper was supported by $\mathrm{NCl}$ grant R01CA217161. Dr. Dashwood was supported by Contract HHSN261201500018I/ HHSN26100004 from the NCI PREVENT Program; NCI grants RO1CA090890, RO1CA122959, and P30CA016672; the John S. Dunn Foundation; and a Chancellor's Research Initiative from Texas A\&M University. Dr. Demehri was supported by NIH grants DP5OD021353 and U01CA233097; the Burroughs Wellcome Fund; the Breast Cancer Alliance; and the ESSCO MGH Breast Cancer Research Fund. Dr. Disis was supported by the: Helen B. Slonaker Endowed Professor for Cancer Research and an American Cancer Society Clinical Professorship. Dr. Johnson was supported by $\mathrm{NCl}$ Contract No. N01CN2015000037 and NCl grant R01CA223804. Dr. Kensler was supported by $\mathrm{NCl}$ grant R35CA197222. Dr. Khan was supported by $\mathrm{NCl}$ Contract HHFN 2612201200035I/HHSN26100003. Dr. Liby's studies were supported NCl grant R01CA226690, Breast Cancer Research Foundation (BCRF-19-096), and Michigan Economic Development Fund (MiTRAC Subk00010972). Dr Lipkin was supported by $\mathrm{NCl}$ grant U01CA233056. Dr. Mallery was supported by NCl grants R01CA211611 and R01CA227273. Dr. Rao was supported by Contract No. N01CN250026 from the NCI PREVENT Program and NCl grant R01CA213987. Dr. Roden was supported by $\mathrm{NCl}$ grants P30CA06973, P50CA098252, R01CA233486, R01CA237067. Dr. Sharp was supported by NCl grant R01CA193835. Dr. Shirwan was supported by NCl grant R41CA199956 and the Kentucky Science and Technology Corporation (KSTC-184-512-16-237). Dr. Siegfried was supported by NCl grant F31CA213982 (PI Christian Njatcha); the Masonic Cancer Center; the State of Minnesota Eagles; and two grants from the University of Minnesota Foundation: the Drug Development Program and the Frederick and Alice Stark Endowment. Dr. Vilar was supported by $\mathrm{NCl}$ grants R01CA219463 and P30 CA016672 and a gift from the Feinberg Family Foundation. Dr. You was supported by the Contract HHSN2612015000371/ HHSN26100005 and 75N91019D00020/75N91019F00130 from the NCI PREVENT Program.

\section{CONFLICTS OF INTEREST}

Drs. Allen, Clapper, Dashwood, Demehri, DuBois, Johnson, Kensler, Khan, Mallery, Miller, Mohammed, Rao, Schoen, Sharp, Szabo, and You have no FCOI to declare. Dr. Brown owns stock in GeneTex, whose products are not discussed in this manuscript. Dr. Chan has previously received grant support and consulting fees from Bayer Pharma AG; consulting fees from Pfizer Inc. and Boehringer Ingelheim. Dr.
Disis received grant funding from Pfizer, Precigen, Bavarian Nordisk, and Veanna; is a stockholder in Epithany; and is an inventor with patents held by the University of Washington. Dr. Glynn has received support from research grants to his employer from AstraZeneca, Kowa, and Pfizer. The Brigham \& Women's Hospital holds patents for use of inflammatory biomarkers in cardiovascular disease. The CANTOS trial was funded by Novartis. Dr. Liby is an inventor on patents for synthetic triterpenoids and rexinoids. Dr. Lipkin is PI on grant U01-CA233056 that includes work performed by Nouscom, LLC. Dr. Meuillet is a scientific co-founder and member of the Management Team of PHusis, which is developing PHT-427. Dr. Roden is a co-founder of and has an equity ownership interest in Papivax LLC. Also, he owns Papivax Biotech Inc. stock options and is a member of Papivax Biotech Inc.'s Scientific Advisory Board. Under a licensing agreement between Papivax Biotech, Inc. and the Johns Hopkins University, the University and Dr. Roden are entitled to royalties on an invention described in this article. This arrangement has been reviewed and approved by the Johns Hopkins University in accordance with its conflict of interest policies. Dr. Siegfried has a US Patent Pending: \#62650892. Dr. Shirwan is a CEO at FasCure Therapeutics, LLC, and has ownership interest (including stocks and patents) in the Company. Dr. Vilar has a consulting or advisory role with Janssen Research and Development, and Recursion Pharma.

\section{ORCID}

Mark Steven Miller, https://orcid.org/0000-0003-1191-0977

Peter J. Allen, https://orcid.org/0000-0001-7912-9197

Powel H. Brown, https://orcid.org/0000-0002-3398-163X

Andrew T. Chan, https://orcid.org/0000-0001-7284-6767

Margie L. Clapper, https://orcid.org/0000-0001-7712-5109

Roderick H. Dashwood,

https://orcid.org/0000-0003-0351-4034

Shadmehr Demehri, https://orcid.org/0000-0002-7913-2641

Mary L. Disis, https://orcid.org/0000-0001-7653-4648

Raymond N. DuBois, https://orcid.org/0000-0002-6385-7154

Robert J. Glynn, https://orcid.org/0000-0002-0697-8996

Thomas W. Kensler, https://orcid.org/0000-0002-6676-261X

Seema A. Khan, https://orcid.org/0000-0002-6307-2023

Bryon D. Johnson, https://orcid.org/0000-0001-7416-3396

Karen T. Liby, https://orcid.org/0000-0003-3317-3437

Steven M. Lipkin, https://orcid.org/0000-0002-0603-9139

Susan R. Mallery, https://orcid.org/0000-0003-3122-0244

Emmanuelle J. Meuillet,

https://orcid.org/0000-0002-2242-0810

Richard B.S. Roden, https://orcid.org/0000-0002-2506-0195

Robert E. Schoen, https://orcid.org/0000-0001-7153-2766

Zelton D. Sharp, https://orcid.org/0000-0002-6580-5366

Haval Shirwan, https://orcid.org/0000-0002-1657-9470

Jill M. Siegfried, https://orcid.org/0000-0002-0887-5448

Chinthalapally V. Rao, https://orcid.org/0000-0002-5715-7979 
Ming You, https://orcid.org/0000-0002-9741-268X

Eduardo Vilar, https://orcid.org/0000-0001-6404-3761

Eva Szabo, https://orcid.org/0000-0003-3891-0942

Altaf Mohammed, https://orcid.org/0000-0003-1058-6909

\section{REFERENCES}

1. Brahme NN, Szabo E. Cancer prevention in the era of precision oncology. Clin Pharmacol Ther 2017;101:575-7.

2. Maresso KC, Tsai KY, Brown PH, Szabo E, Lippman S, Hawk ET. Molecular cancer prevention: current status and future directions. CA Cancer J Clin 2015;65:345-83.

3. Mohammed A, Fox JT, Miller MS. Cancer chemoprevention: preclinical in vivo alternate dosing strategies to reduce drug toxicities. Toxicol Sci 2019;170:251-9.

4. Wojtowicz ME, Dunn BK, Umar A. Immunologic approaches to cancer prevention-current status, challenges, and future perspectives. Semin Oncol 2016;43:161-72.

5. Siegel RL, Miller KD, Jemal A. Cancer statistics, 2019. CA Cancer J Clin 2019;69:7-34.

6. Finn OJ. The dawn of vaccines for cancer prevention. Nat Rev Immunol 2018;18:183-94.

7. Candeias SM, Gaipl US. The immune system in cancer prevention, development and therapy. Anticancer Agents Med Chem 2016;16:101-7.

8. Cen B, Lang JD, Du Y, Wei J, Xiong Y, Bradley N, et al. Prostaglandin $\mathrm{E}_{2}$ induces miR675-5p to promote colorectal tumor metastasis via modulation of p53 expression. Gastroenterology 2020;158:971-84.e10.

9. Wang $D$, DuBois RN. Role of prostanoids in gastrointestinal cancer. J Clin Invest 2018;128:2732-42.

10. Liby KT, Sporn MB. Rexinoids for prevention and treatment of cancer: opportunities and challenges. Curr Top Med Chem 2017;17:721-30.

11. Liby KT, Sporn MB. Synthetic oleanane triterpenoids: multifunctional drugs with a broad range of applications for prevention and treatment of chronic disease. Pharmacol Rev 2012;64:972-1003.

12. Zhang D, Rennhack J, Andrechek ER, Rockwell CE, Liby KT. Identification of an unfavorable immune signature in advanced lung tumors from Nrf2-deficient mice. Antioxid Redox Signal 2018;29:1535-52.

13. Hasty P, Livi CB, Dodds SG, Jones D, Strong R, Javors M, et al. eRapa restores a normal life span in a FAP mouse model. Cancer Prev Res (Phila) 2014;7:169-78.

14. Parihar M, Dodds SG, Hubbard G, Javors MA, Strong R, Hasty $\mathrm{P}$, et al. Rapamycin extends life span in $\mathrm{Apc}^{\mathrm{Min} /+}$ colon cancer FAP model. Clin Colorectal Cancer 2021;20:e61-70.

15. Yagishita Y, Fahey JW, Dinkova-Kostova AT, Kensler TW. Broccoli or sulforaphane: is it the source or dose that matters? Molecules 2019;24:3593.

16. Yagishita Y, Gatbonton-Schwager TN, McCallum ML, Kensler TW. Current landscape of NRF2 biomarkers in clinical trials. Antioxidants (Basel) 2020;9:716.
17. Egner PA, Chen JG, Zarth AT, Ng DK, Wang JB, Kensler KH, et al. Rapid and sustainable detoxication of airborne pollutants by broccoli sprout beverage: results of a randomized clinical trial in China. Cancer Prev Res (Phila) 2014;7:813-23.

18. Chen JG, Johnson J, Egner P, Ng D, Zhu J, Wang JB, et al. Dose-dependent detoxication of the airborne pollutant benzene in a randomized trial of broccoli sprout beverage in Qidong, China. Am J Clin Nutr 2019;110:675-84.

19. Ridker PM, Everett BM, Thuren T, MacFadyen JG, Chang WH, Ballantyne C, et al. Antiinflammatory therapy with canakinumab for atherosclerotic disease. N Engl J Med 2017;377:1119-31.

20. Ridker PM, MacFadyen JG, Thuren T, Everett BM, Libby P, Glynn RJ. Effect of interleukin-1 $\beta$ inhibition with canakinumab on incident lung cancer in patients with atherosclerosis: exploratory results from a randomised, double-blind, placebo-controlled trial. Lancet 2017;390:1833-42.

21. Valeri L, VanderWeele TJ. SAS macro for causal mediation analysis with survival data. Epidemiology 2015;26:e23-4.

22. Sharma RK, Elpek KG, Yolcu ES, Schabowsky RH, Zhao H, Bandura-Morgan L, et al. Costimulation as a platform for the development of vaccines: a peptide-based vaccine containing a novel form of 4-1BB ligand eradicates established tumors. Cancer Res 2009;69:4319-26.

23. Schabowsky RH, Elpek KG, Madireddi S, Sharma RK, Yolcu ES, Bandura-Morgan L, et al. A novel form of 4-1BBL has better immunomodulatory activity than an agonistic anti-4-1BB Ab without Ab-associated severe toxicity. Vaccine 2009;28:512-22.

24. Barsoumian HB, Batra L, Shrestha P, Bowen WS, Zhao H, Egilmez NK, et al. A novel form of 4-1BBL prevents cancer development via nonspecific activation of $\mathrm{CD} 4^{+} \mathrm{T}$ and natural killer cells. Cancer Res 2019;79:783-94.

25. Demehri S, Cunningham TJ, Manivasagam S, Ngo KH, Moradi Tuchayi S, Reddy R, et al. Thymic stromal lymphopoietin blocks early stages of breast carcinogenesis. J Clin Invest 2016;126:1458-70.

26. Mohammed A, Janakiram NB, Madka V, Zhang Y, Singh A, Biddick $L$, et al. Intermittent dosing regimens of aspirin and naproxen inhibit azoxymethane-induced colon adenoma progression to adenocarcinoma and invasive carcinoma. Cancer Prev Res (Phila) 2019;12:751-62.

27. Amos-Landgraf JM, Kwong LN, Kendziorski CM, Reichelderfer $\mathrm{M}$, Torrealba J, Weichert J, et al. A target-selected Apc-mutant rat kindred enhances the modeling of familial human colon cancer. Proc Natl Acad Sci USA 2007;104:4036-41.

28. Ertem F, Dashwood WM, Rajendran P, Raju G, Rashid A, Dashwood R. Development of a murine colonoscopic polypectomy model (with videos). Gastrointest Endosc 2016;83:1272-6.

29. Ertem FU, Zhang W, Chang K, Mohaiza Dashwood W, Rajendran P, Sun D, et al. Oncogenic targets Mmp7, S100a9, $\mathrm{Nppb}$ and Aldh1a3 from transcriptome profiling of FAP and Pirc adenomas are downregulated in response to tumor suppression by Clotam. Int J Cancer 2017;140:460-8.

30. Okonkwo A, Mitra J, Johnson GS, Li L, Dashwood WM, Hegde 
ML, et al. Heterocyclic analogs of sulforaphane trigger DNA damage and impede DNA repair in colon cancer cells: interplay of HATs and HDACs. Mol Nutr Food Res 2018;62:e1800228.

31. Rajendran P, Johnson G, Li L, Chen YS, Dashwood M, Nguyen $\mathrm{N}$, et al. Acetylation of CCAR2 establishes a BET/BRD9 acetyl switch in response to combined deacetylase and bromodomain inhibition. Cancer Res 2019;79:918-27.

32. Damiani E, Duran MN, Mohan N, Rajendran P, Dashwood RH. Targeting epigenetic 'readers' with natural compounds for cancer interception. J Cancer Prev 2020;25:189-203.

33. Reyes-Uribe L, Wu W, Gelincik O, Bommi PV, FranciscoCruz A, Solis LM, et al. Naproxen chemoprevention promotes immune activation in Lynch syndrome colorectal mucosa. Gut 2021;70:555-66.

34. Kucherlapati MH, Lee K, Nguyen AA, Clark AB, Hou H Jr, Rosulek A, et al. An Msh2 conditional knockout mouse for studying intestinal cancer and testing anticancer agents. Gastroenterology 2010;138:993-1002.e1.

35. Burn J, Sheth H, Elliott F, Reed L, Macrae F, Mecklin JP, et al. Cancer prevention with aspirin in hereditary colorectal cancer (Lynch syndrome), 10-year follow-up and registry-based 20-year data in the CAPP2 study: a double-blind, randomised, placebocontrolled trial. Lancet 2020;395:1855-63.

36. Pan J, Zhang Q, Palen K, Wang L, Qiao L, Johnson B, et al. Potentiation of Kras peptide cancer vaccine by avasimibe, a cholesterol modulator. EBioMedicine 2019;49:72-81.

37. Pan J, Zhang Q, Sei S, Shoemaker RH, Lubet RA, Wang Y, et al. Immunoprevention of KRAS-driven lung adenocarcinoma by a multipeptide vaccine. Oncotarget 2017;8:82689-99.

38. Cecil DL, Holt GE, Park KH, Gad E, Rastetter L, Childs J, et al. Elimination of IL-10-inducing T-helper epitopes from an IGFBP-2 vaccine ensures potent antitumor activity. Cancer Res 2014;74:2710-8.

39. Cecil DL, Slota M, O'Meara MM, Curtis BC, Gad E, Dang Y, et al. Immunization against HIF-1 $\alpha$ inhibits the growth of basal mammary tumors and targets mammary stem cells in vivo. Clin Cancer Res 2017;23:3396-404

40. Broussard EK, Kim R, Wiley JC, Marquez JP, Annis JE, Pritchard $\mathrm{D}$, et al. Identification of putative immunologic targets for colon cancer prevention based on conserved gene upregulation from preinvasive to malignant lesions. Cancer Prev Res (Phila) 2013;6:666-74.

41. Willis JA, Reyes-Uribe L, Chang K, Lipkin SM, Vilar E. Immune activation in mismatch repair-deficient carcinogenesis: more than just mutational rate. Clin Cancer Res 2020;26:11-7.

42. Drew DA, Schuck MM, Magicheva-Gupta MV, Stewart KO, Gilpin KK, Miller P, et al. Effect of low-dose and standard-dose aspirin on $\mathrm{PGE}_{2}$ biosynthesis among individuals with colorectal adenomas: a randomized clinical trial. Cancer Prev Res (Phila) 2020;13:877-88.

43. McNeil JJ, Gibbs P, Orchard SG, Lockery JE, Bernstein WB, Cao Y, et al. Effect of aspirin on cancer incidence and mortality in older adults. J Natl Cancer Inst 2021;113:258-65. 